\title{
FACIAL IMAGE CLASSIFICATION AND SEARCHING -A SURVEY
}

\author{
Dr.S.Vijayarani ${ }^{1}$ and Mrs.M.Vinupriya ${ }^{2}$
}

1 Assistant Professor, Department of Computer Science, School of Computer Science and Engineering, Bharathiar University, Coimbatore -641 046, Tamil Nadu

2 Research Scholar, Department of Computer Science, School of Computer Science and Engineering, Bharathiar University, Coimbatore - 641 046, Tamil Nadu

\begin{abstract}
Recent developments in the area of image mining have shown the way for incredible growth in extensively large and detailed image databases. The images which are available in these databases, if checked, can endow with valuable information to the human users. As one of the most successful applications of image analysis and understanding, face recognition has recently gained important attention particularly throughout the past many years. Though tracking and recognizing face objects is a routine task, building such a system is still an active research. Among several proposed face recognition schemes, shape based approaches are possibly the most promising ones. This paper provides an overview of various classification and retrieval methods that were proposed earlier in literature. Also, this paper provides a marginal summary for future research and enhancements in face detection.
\end{abstract}

\section{KEYWORDS}

Image mining, face detection, classification, retrieval, SVM technique, searching.

\section{INTRODUCTION}

Data mining is a process which uses a mixture of data analysis tools to discover patterns and relationships in data that may be used to make valid predictions. It permits users to analyze data from many different dimensions or angles, classify it, and summarize the relationships identified. Technically, data mining is the method of identifying correlations or patterns among dozens of fields in massive relative databases. Some of the research areas in data mining are web mining, text mining, data streams, image mining, sequence mining and multimedia mining. Image mining facilitates the abstraction of hidden information which is not clearly accrued in the image. It is used to detect unfamiliar patterns and abstract inherent and useful data from images stored in the large databases. Therefore image mining deals with making relationships between different images from large image databases. A large amount of characteristics used for image category are: shape, texture, color and spatial features.

DOI : $10.5121 /$ ijitmc.2014.2203 
Image mining is not only a limb of data mining and knowledge discovery, but also an highly developed research area which includes digital image processing, image indexing and retrieval, image understanding, database, artificial intelligence, pattern discovery and so on[7].

Research in image mining can be broadly classified in two main directions [6] Domain- specific applications where the focus is in the process of extracting the most applicable image features into a form appropriate for data mining.

(2) General applications where the focus is to create the image patterns that may be helpful in the understanding of the communication

between high-level human perceptions of image and low level image features. Both are used to extract most relevant image feature and later to generate image patterns. A number of image mining systems have been developed for different applications which include remote sensing, natural scene recognition, weather forecasting, egeria detection, criminal investigation, image segmentation, medical diagnosis, space research, biology, image classification and retrieval etc.

Face Recognition System is a computer application for automatically identifying or verifying a person from a digital image or a single frame from a video source. This can be done by comparing selected facial characteristics of the likeness and a facial database. It executes that by comparing the face of the accessing user with a database of faces previously stored in memory. Face recognition presents a challenging problem in the area of image analysis and computer vision, and it has received a great deal of interest over the last few years because of its applications in different domains. There are many problems that exist due to many factors that can affect the images. When processing images one must take into account the variations in lightweight, image quality, the individuals pose and facial expressions along with others.

The rest of the paper is organized as follows. Section 2 describes the different approaches of image based face recognition and ITS applications. Section 3 discusses about the importance of classification and searching in facial images. Section 4 provides a literature survey. Section 5 shows various research directions involved in the face recognition and conclusion is given in Section 6.

\section{DIFFERENT APPROACHES AND APPLICATIONS IN FACE RECOGNITION}

Different types of facial images are taken for face detection. The images that are collected in a semi-controlled environment are used as input. Images were taken in uncontrolled indoor environment and different facial expressions (such as open / closed eyes, smiling / not smiling) or configurations (such as w/glasses, center-light, happy, left-light, normal, right-light, w/no glasses, sad, sleepy, surprised, and wink). The images are taken by varying the lighting and facial details (glasses / no glasses) at different times and are used as input. 


\subsection{Different approaches}

There are numerous ways to detect a face in a scene and it is easier and harder ones. Here is a list of the most familiar approaches in face detection:

$>$ Identifying faces in images with prohibited background

$>$ Identifying faces by motion

$>$ Using a combination of the above

$>$ Identifying faces in unconstrained scenes

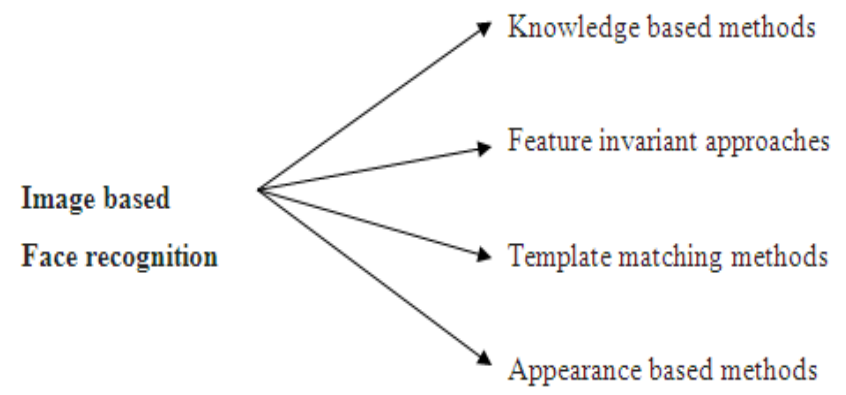

Figure 1: Taxonomy of Iamge based Face Recognition Approaches

The existing techniques are reviewed to detect faces from a single intensity or color image. Single image detection methods are classified into four categories [11],

1. Knowledge-based methods. These rule-based procedures encode human information of what constitutes a distinctive face. Usually, the rules confine the relationships between facial features. These procedures are designed mainly for face localization. Knowledge based methods can be studied by two different approaches; which are top-down methods and bottom-up methods.

2. Feature invariant approaches. These algorithms intend to find structural features that live even when the pose, viewpoint, or lighting conditions differ, and then use these to find faces. These methods are designed mainly for face localization.

3. Template matching methods. There are number of standard patterns of a face are stored which is used to describe the face as a whole or the facial features separately. The associations between an input image and the stored patterns are worked out for detection. These methods have been applied for both face localization and detection. Template matching researches can be divided into two subcategories which are, researches using predefined templates and the other is researches using deformable templates.

4. Appearance-based methods. In difference to template matching, the models (or templates) are first learned from a set of training images which should capture the representative variability of facial appearance. These learned models are 
then utilised for detection. These methods are designed largely for face detection. There are number of approaches using the appearance based methods are, eigenfaces, distribution-based methods, neural networks, support vector machines, sparse network of winnows, Naïve Bayes classifiers, hidden Markov model , information theoretical approach and inductive learning.

\subsection{APPLICATIONS}

Face recognition is utilised for two primary jobs:

1. Verification (one-to-one matching): When offered with a face image of an unidentified individual along with a claim of individuality, ascertaining whether the individual is who he/she claims to be.

2. Identification (one-to-many matching): When a system offered with an image of an unidentified individual, determining that person's identity by matching (possibly after encoding) that image with a database of (possibly encoded) images of identified individuals.

There are several application areas in which face recognition can be exploited for these two reasons, a few of which are outlined below.

Security: It is mostly utilised for accessing controls to airports/seaports, buildings, airports/seaports, ATM machines and border checkpoints [8]; computer/ network security [4]; email authentication on multimedia workstations.

Surveillance: It can be seen with a large number of CCTVs can be examined to look for identified criminals, drug offenders, etc. and authorities can be notified when one is to be found; for instance, this procedure was used at the Super Bowl 2001 game at Tampa, Florida [3];

$>$ General identity verification is done for electoral registration, electronic commerce, banking, identifying newborns, passports, national IDs, drivers' licenses, workers IDs, etc.

$>$ Criminal justice systems such as mug-shot/booking systems, post-event investigation, forensics.

$>$ Image database investigations: used for searching image databases of licensed drivers, promote recipients, immigrants, missing children and police bookings.

$>$ "Smart Card" applications: In the maintenance of facial images database, the face-print

can be stored in a bar code, smart card or magnetic stripe, authentication of which is achieved by matching the live image and the retained template [18]. 
Multi-media environments with adaptive individual computer interfaces a part of ubiquitous or context aware systems, behavior examining at childcare or old people's centers, identifying a customer and assessing his needs $[16,8]$.

\section{FACIAL IMAGE CLASSIFICATION AND SEARCHING}

Image classification intends to find a description that can best describe the images in one class and to distinguish these images from all the other classes. It is one of the most often used methods for extracting information from images. Classification involves verdict rules that partition the data into disjoint groups. Training data set is the input for the classification data set, whose class labels are already known. In Classification, usually several features are used for a set of pixels i.e., many images of a particular object are needed. Image search engines are currently dependent on textual metadata. This information can be in the form of filenames, images, manual annotations, or surrounding text. However, for the huge bulk of images on the internet and in peoples' private collections, this data is often confusing, erroneous, or simply not present. This presents a great opportunity to use attribute classifiers on images with faces, thereby making them searchable. An image retrieval system is a computer system which is used for searching, browsing and retrieving images from large set of databases. Many image retrieval systems both business and research have been built. A large amount of image retrieval systems support one or more of the following choices:

$>$ Random browsing

$>$ Searching through example

$>$ Searching through sketch

$>$ Searching through text (including key word or speech)

$>$ Steering with modified image categories.

\section{RELATED WORKS}

Several researches have been carried on this face detection. This section presents a study on various classification and retrieval methods that were proposed earlier.

S.Ravi, S.Wilson [15] proposed a color model conversion algorithm based on chrominance color, this information is used for detecting face region. The facial features such as eyes, nose, and mouth are used to find the region of the feature pixel by applying threshold measurements. The algorithm provides a better accuracy for classifying the gender using SVM classification algorithm. The main issue involved is choosing the correct threshold parameter for facial feature detection and gender classification, which clearly might be inadequate for the data.

Lin-Lin Huanga, AkinobuShimizu, Yoshihoro Hagihara, Hidefumi Kobatake [9], proposed a classification-based approach for locating frontal and nearly frontal faces in cluttered images. The directional decomposition of gradient provides a better discrimination ability 
than the image intensity and the gradient map, and it was shown that the combination of gradient

direction and intensity further improves the detection performance. To improve the detection performance, gradient direction features from local window images are extracted as input for underlying two-class classifier. Simple and complex background images are taken to check the performance and it provides a better performance.

Yongmin Li, Shaogang Gong and Heather Liddell [19], discussed about the SVM approach used for multi-view face detection and recognition. It involves the following methods

1.SVM construct pose estimators which gives fast estimation of head pose. 2. Symmetrical property of the face images are used to classify the half possible views with the trained set. 3. The improvement of detection accuracy and easy computation, pose information is used to guide the selection of face detectors by using pose change smoothing technique.

Saman cooray, Noel O' Connor [14], has discussed to detect frontal faces; the author had used a technique that combines feature extraction and statistical face classification. The use of eye facial feature points enables to derive a normalized search space by eliminating the requirement of analyzing the image at multiple scales for detecting different sized faces. Since the search space is normalized, the need for analyzing the image at each image pixel location is eliminated. This in turn provides a promising approach for facial classification.

Bo WU, Haizhou AI and Chang HUANG [2], described a LUT weak classifier based boosting method for face retrieval by demographic classification. A Haar feature based 2D LUT-type weak classifier was developed for multi-class problems and had used a variation of boosting algorithm for multi-class multilabel problems, Adaboost.MH to learn the demographic classifiers. A model of automatic demographic face retrieval system is presented and the experimental results show its potentials in the management of a large facial image database for online retrieval applications.

Ahmed Abdu Alattab, Sameem Abdul Kareem [1]: has discussed about the integration of verbal description of human face and eigen face feature achieved excellent results in the retrieval of face image when compared to retrieval by image content that reduces the semantic gap between high level query requirement represented by user verbal description and low level facial features represented by image content features. Combining the two methods of query by description and query by image example, the accuracy of the retrieval process is improved automatically and the needed time to find the desired faces is also minimized. The idea was based on matching the user verbal description of the query face with the annotated description of the faces in the database. The system then uses eigen faces features for further searches on narrowed down search space of the pruned set facial images, achieving more accurate results.

Yogesh R. Tayade1, Prof. S.M. Bansode2 [18], developed an efficient method for face retrieval by merging three different algorithms SIFT, LBP and IDSC. An image is given as an input, it is filtered and it is represented in a sparse matrix that derives SIFT and LBP 
features. Through this work, the face images are successfully retrieved from the trained dataset of Labeled Faces in Wild (LFW) images which competently achieves better retrieval rate.

Yannis Avrithis, Nicolas Tsapatsoulis and Stefanos Kollias [17], portrays that to extract objects from images color segmentation has proved to be a powerful tool, especially in the case of human faces that are usually characterized by uniform color. The M-RSST algorithm eliminates facial details and provides a single object for each face. Moreover, chrominance components provided with a probabilistic model are used in an efficient way for retrieving facial images from image databases. The interactive form of the proposed system adapts the model to the needs of the user and consequently leads to much more meaningful retrieval results.

Neeraj Kumar, et al., [12], discussed about how to automatically train classifiers for describable aspects of visual appearance - attributes and similes. These classifiers are learned using huge collections of labeled images obtained from the internet. They demonstrated the use of these describable attributes for performing face verification and image search. It was performing better in aspects such as, attribute classification, face verification, and search (qualitatively).

\section{RESEARCH DIRECTIONS}

The challenges combined with face detection can be attributed to the following factors

$[8,4,10]:$

1. Pose: The images of a face vary due to the relative camera-face pose (frontal, 45 degree, profile, upside down), and some facial characteristics such as an eye or the nose may become partly or wholly occluded.

2. Presence or absence of structural elements: Facial characteristics such as beards, mustaches, and glasses may or may not be present and there is a great deal of variability among these components including color, shape, and size.

3. Facial expression. The appearances of faces are straightly influenced by a person's facial expression.

4. Occlusion. Faces may be partly occluded by other objects. An image with a group of people, some faces may partly occlude other faces.

5. Image orientation: Face images straightly differ for different rotations about the camera's optical axis.

6. Imaging conditions: When the image is produced, features such as lighting (spectra, source distribution and intensity) and camera characteristics (sensor response, lenses) affects the outer shell of a face.

7. The age: As the face matures, it modifies some of its most enduring properties (e.g., shape of cranium) and acquires new attributes (e.g., wrinkles,spot). These changes gives the basic information about the aging of the face.

8. Changes in illumination: Person face was slower and less accurate at matching and naming faces when there was a change in illumination direction. 
9. Forensic Sketch Recognition: Sketches can be drawn from human memory when no image is available. For example when most terrible of crimes committed such as murder, sexual assualt, etc..

10. Component-based face recognition: Perform face matching and image retrieval per facial component such as eyes, nose, mouth, eye brows, chin. It promotes partial face matching and individuality models.

\section{CONCLUSION}

Face recognition technologies have been connected generally with very costly top secure applications. Beside with all the biometric organisms, face recognition approach possesses one vast advantage, which is its user-friendliness. This paper presents a survey on various face detection techniques and algorithms that was proposed earlier by researchers for the better development in the field of classification and content based image retrieval. It also provides an overview of some of the ongoing researches in face detection. Face detection in entirely unrestrained settings remains a very challenging task, particularly due to the major pose and lighting variations.

\section{REFERENCES}

[1] Ahmed Abdu Alattab, Sameem Abdul Kareem Facial Image Retrieval Based on Eigen faces and Semantic Features Proc. of the International Conference on Advances in Computer and Information Technology - ACIT 2012

[2] Bo WU, Haizhou AI and Chang HUANG Facial Image Retrieval Based on Demographic Classification

[3] D. McCullagh, "Call It Super Bowl Face Scan 1," in Wired Magazine, 2001.

[4] J. N. K. Liu, M. Wang, and B. Feng, "iBotGuard: "an Internet-based intelligent robot security system using invariant face recognition against intruder," IEEE Transactions on Systems Man and Cybernetics Part C-Applications and Reviews, Vol.35, pp.97-105, 2005.

[5] Jawad Nagi, Syed Khaleel Ahmed Farrukh Nagi A MATLAB based Face Recognition System using Image Processing and Neural Networks 4th International Colloquium on Signal Processing and its Applications, March 7-9, 2008, Kuala Lumpur, Malaysia. ISBN: 978-983-42747-9-5

[6] Ji Zhang Wynne Hsu Mong Li Lee "Image Mining: Trends and Developments".

[7] Ji Zhang Wynne Hsu Mong Li Lee "Image Mining: Issues, Frameworks and Techniques" Proceedings of the Second International Workshop on Multimedia Data Mining (MDM/KDD 2001) Proceedings of the Second International Workshop on Multimedia Data Mining (MDM/KDD'2001), in conjunction with ACM SIGKDD conference. San Francisco, USA, August 26, 2001.

[8] Kwangbaek Kim Intelligent Immigration Control System by Using Passport Recognition and Face Verification ISNN 2005, LNCS 3497, pp. 147-156, 2005. (C) Springer-Verlag Berlin Heidelberg 2005.

[9] Lin-Lin Huanga; AkinobuShimizu a, Yoshihoro Hagiharab, Hidefumi Kobatakea Gradient feature extraction for classi"cation-based face Detection Pattern Recognition 36 (2003) 2501 - 2511 Pattern Recognition Society. Published by Elsevier Ltd.

[10] Manisha Deorao Urkude Dr. S.B. Kishor Dr. Sunil Gulabrao Naranje "Study of Open Challenges in Face Recognition and Expected Result "Indian Journal of Applied Research Volume: 3 | Issue: 6 | June 2013 | ISSN - 2249-555X

[11] Ming-Hsuan Yang, Member, IEEE, David J. Kriegman, Senior Member, IEEE, and Narendra Ahuja, Fellow, IEEE "Detecting Faces in Images: A Survey "IEEE Transaction On Pattern Analysis And Machine Intelligence, vol. 24, no. 1, January 2002 
[12] Neeraj Kumar, Student Member, IEEE, Alexander C. Berg, Member, IEEE, Peter N. Belhumeur, and Shree K. Nayar, Member, IEEE "Describable Visual Attributes for Face Verification and Image Search" IEEE Transactions On Pattern Analysis And Machine Intelligence.

[13] Rabia Jafri* and Hamid R. Arabnia* "A Survey of Face Recognition Techniques" Journal of Information Processing Systems, Vol.5, No.2, June 2009

[14] Saman cooray, Noel O' Connor Facial features and appearance-based classification for face detection in color images.

[15] S.Ravi , S.Wilson Face Detection with Facial Features and Gender Classification Based On Support Vector Machine 2010 Special Issue - International Journal of Imaging Science and Engineering

[16] T. Choudhry, B. Clarkson, T. Jebara, and A. Pentland, "Multimodal person recognition using unconstrained audio and video," in Proceedings, International Conference on Audio and Video-Based Person Authentication, 1999, pp.176-181

[17] Yannis Avrithis, Nicolas Tsapatsoulis and Stefanos Kollias Color-Based Retrieval of Facial Images. [18] Yogesh R. Tayade1, Prof. S.M. Bansode2 An Efficient Face Recognition and Retrieval Using LBP and SIFT International Journal of Advanced Research in Computer and Communication Engineering Vol. 2, Issue 4, April 2013

[19 ]Yongmin Li, Shaogang Gong and Heather Liddell Support Vector Regression and Classification Based Multi-view Face Detection and Recognition.

[20] Zeynep Orman, Abdulkadir Battal and Erdem Kemer A Study on Face, Eye Detection and Gaze Estimation International Journal of Computer Science \& Engineering Survey (IJCSES) Vol.2, No.3, August 2011.

\section{Authors}

Dr. S.Vijayarani has completed MCA, M.Phil and Ph.D in Computer Science. She is working as Assistant Professor in the School of Computer Science and Engineering, Bharathiar University, Coimbatore. Her fields of research interest are data mining, privacy, security, bioinformatics and data streams. She has published papers in the international journals and presented research papers in international and national conferences.

Mrs. M.Vinupriya has completed MCA. She is currently pursuing her M.Phil in Computer Science in the School of Computer Science and Engineering,Bharathiar University, Coimbatore. Her fields of interest are Image Mining in data mining and privacy preserving in Data mining.
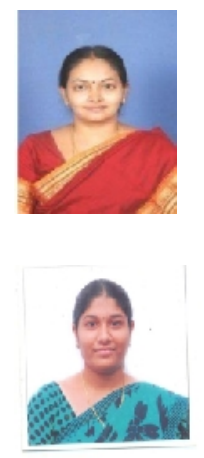